\title{
Statistical Analysis of Blood- to Breath-Alcohol Ratio Data in the Logarithm-Transformed and Non-Transformed Modes
}

\author{
Dominick A. Labianca and G. Simpson
}

Department of Chemistry, Brooklyn College of The City University of New York, Brooklyn, N. Y., USA

Summary: The statistical analysis of non-transformed and logarithm-transformed blood- to breath-alcohol ratios ("blood/breath ratios") is detailed. The data analyzed were derived from 137 simultaneous blood-alcohol and breathalcohol concentration measurements made between 15 and $179 \mathrm{~min}$ after the end of drinking, with 136 of the measurements obtained during the 15- to 124-min time frame. Although the distribution of the non-transformed ratios is positively skewed, and that of the logarithm-transformed data more closely approximates the normal distribution upon visual inspection, both analyses generated results that do not differ significantly from each other when considered in the context of "mean ratios $\pm 2 S D$ ". This is in accord with the results of the KolmogorovSimirnov goodness-of-fit test, which does not reject either dataset and demonstrates that both are approximately normal. Since the logarithm-transformed data generate more conservative statistical blood/breath ratio ranges than the non-transformed data, they were selected as the basis for the principal conclusion of this work. That conclusion is a refutation of the argument that, breath-alcohol analyzers relying on a $2100: 1 \mathrm{blood} / \mathrm{breath}$ ratio tend to underestimate the blood-alcohol concentrations of driving-while-intoxicated arrestees because the commonly accepted mean postabsorptive ratio is $2300: 1$. In fact, whenever the absorption status of a driving-while-intoxicated arrestee at the time of a breath test cannot be definitively established, the results of this work support the application of a relative error range of $-40 \%$ to $+28 \%$ for $95 \%$ of the population, based on a statistical blood/breath ratio range of $1259: 1$ to $2679: 1$, and $-46 \%$ to $+42 \%$ for $99 \%$ of the population, based on a statistical range of $1128: 1$ to $2989: 1$.

\section{Introduction}

Many biological measurements do not conform to normal error analysis, so that the value of the normal error curve as a descriptive statistic has been challenged $(1: 112)$. Frequently, however, the initial measurements of a particular random variable may be normally distributed on a different scale, and, as emphasized by Gaddum (2), this would result in "an increase in the accuracy and scope of the conclusions drawn from [the measurements]."

An application of this argument was endorsed by Jones (3) in 1989 in connection with the calculation of blood/ breath ratios from measurements of two random variables, namely blood-alcohol concentrations (BACs) and breath-alcohol concentrations (BrACs). Jones recommended that blood/breath ratio data be evaluated on a logarithmic scale, specifically logarithm to base 10 . Under these circumstances, if the logarithm of the original variate - in this case, $\log R$, where $R$ denotes the original blood/breath ratio variate - is distributed normally, the distribution would be classified lognormal. The use of such a transformation for scientific observations dates back to the work of Galton (4), and its characteristics and applications have been detailed in a monograph by Aitchison \& Brown (5).
The rationale for Jones' recommendation was that, while the ratios of blood-alcohol to breath-alcohol concentrations may not be normally distributed, the differences between these two varibles on an appropriate scale could very well be, as per the argument of Finney (6), whom he cited. Accordingly, the use of the logarithmic transformation would apply because the logarithm of a ratio reflects the difference of the logarithms of the two components of the ratio, as can be confirmed from equations 1 and 2.

$$
\frac{\mathrm{BAC}}{\mathrm{BrAC}}=\mathrm{R}
$$

Consequently,

$$
\log \mathrm{BAC}-\log \mathrm{BrAC}=\log \mathrm{R}
$$

To ascertain the extent to which statistical evaluation of logarithm-transformed blood/breath ratios produces results differing from those generated by the evaluation of the corresponding non-transformed ratios, we have analyzed the blood/breath ratio data of Giguiere \& Simpson (7). We have chosen to focus on this work because it was designed specifically to emphasize the determination of blood-breath ratios during the first 1 to $2 \mathrm{~h}$ after the end of drinking. This is a significant time frame because very few documented studies have employed it. 
Those that have done so have used a limited number of test subjects $(8,9)$ and/or have not tabulated blood/ breath ratio data so that it could be subjected to the type of analysis described here (10). Moreover, this time frame is particularly critical in the legal arena because it impacts on the state of alcohol absorption of the drinker, and it can characterize the motor vehicle operator suspected of driving-while-intoxicated who is routinely evaluated within 1 to $2 \mathrm{~h}$ after the end of drinking via breath-alcohol testing based on a constant blood/ breath ratio, typically $2100: 1$. Given that the generally accepted mean postabsorptive blood/breath ratio is about $2300: 1(11,12)$, and that, therefore, the argument has been offered that breath-alcohol analyzers using the $2100: 1$ conversion tend to underestimate the blood-alcohol concentrations of driving-while-intoxicated arrestees, we consider the statistical analysis of the data of Giguiere \& Simpson (7) to be crucial to assessing the validity of that argument.

\section{Materials and Methods}

The details of Giguiere \& Simpson's experimental protocol, including descriptions of test subjects, ethanol administration, blood sampling, and the analytical methods and instruments used; have been presented elsewhere (7). Therefore, only a brief summary is provided here.

A total of $137 \mathrm{blood} / \mathrm{breath}$ ratios was determined. These were derived from essentially simultaneous blood-alcohol and breath-alcohol concentration measurements conducted on 79 healthy adults ( 73 males and 6 females ranging in age from 19 to 68 years) between 15 and 179 min after the end of drinking on an empty stomach. Of these measurements, the first 136 were made between 15 and $124 \mathrm{~min}$ after the end of drinking. The test group was comprised of drivers who had records of arrest for driving-while-intoxicated and who had volunteered to participate in a blood/breath ratio study. Blood samples were analyzed via gas chromatographic analysis, and breath samples were analyzed via infrared spectrophotometry, using Intoxilyzer models 4011 A, 4011 AS, and 5000 (CMI Inc., Minturn, CO, and, currently, Owensboro, KY).

The data analysis for this work, with the exception of the Geary kurtosis test (13), was done using the StatView 4.5 statistics program for the Macintosh line of computers (Abacus Concepts, Berkeley, CA). Geary's measure of kurtosis is given by his test statistic, $a$, and was calculated using equation 3 . In this equation - and elsewhere in this article - the standard abbreviation "SD" is used to denote the standard deviation instead of " $s$ ". which was used by Geary and which appears in many statistical texts; $x_{i}$ denotes an individual value of either $\mathrm{R}$ or $\log \mathrm{R}$; and, correspondingly, $\bar{x}$ denotes either $\bar{R}$ (mean $R$ ) or $\log \overline{\mathrm{R}}_{\mathrm{G}}$ (logarithm of the geometric mean, $\bar{R}_{G}$, where $\log \bar{R}_{G}$ equals the arithmetic mean of the logarithms of the individual $R s$ ).

$$
a=\frac{\sum_{i=1}^{n}\left|x_{i}-\bar{x}\right|}{\mathrm{SD} \sqrt{n(n-1)}}
$$

The expected value of $a$ in a normal population is $\sqrt{2 / \pi}=0.7979$. Smaller values indicate leptokurtosis (characteristic of peaked distributions with long tapering tails), and larger values show platykurtosis (indicative of flat-topped distributions with short tails) (14). Critical values of $a$ for random samples from a normally distributed population were tabulated by Geary (13), and a more thorough compilation is provided by Pearson \& Hartley (15). Specifically, for $n=137$, critical values of $a$ were obtained by linear interpolation on tabulated values (15) - as described by Snedecor \&
Cochran $(16: 541)$ - for two-sided tests at $2 \%, 10 \%$, and $20 \%$ levels of significance. Thus, for example, the acceptance region for a two-sided test at the $20 \%$ level of significance is dictated by the inquality: $a_{0.10}<a_{\text {Experimental Data }}<a_{0.90}$.

Similar inequalities were employed to assess the skewness $\left(g_{1}\right)$ and kurtosis $\left(g_{2}\right)$ test statistics provided by StatView 4.5 at the $2 \%$, $10 \%$, and $20 \%$ levels of significance (twołsided), using linear interpolation on tabulated critical values $(17: 326)$ to obtain the values corresponding to $n=137$. With regard to $g_{2}$, StatView 4.5 yielded results in accord with the equation for kurtosis $(16: 87)$ that were adjusted by an addition of 3 - which is the expected value for a normal distribution - in order to facilitate comparisons with tabulated critical values. Thus, both values of $g_{2}$ and $g_{2}+3$ are reported in this article. In addition, negative kurtosis is indicated by negative values of $g_{2}\left(g_{2}+3<3\right.$, platykurtosis), and positive kurtosis by positive values of $g_{2}\left(g_{2}+3>3\right.$, leptokurtosis). This pattern is just the reverse of the pattern characterizing the Geary kurtosis test, although when applied to the same data, $a$ and $g_{2}$ usually produce the same conclusions $(16: 88)$.

In addition to the measures of skewness and kurtosis described above, the Kolmogorov-Smirnov normality test $(18-20)$ was also applied to the data of this work to determine if they deviate from a normal distribution. This test is characterized by the KolmogorovSnirnov statistic, D, which represents the maximum absolute difference between the sample cumulative distribution and the target cumulative distribution, which, for this work, is the normal distribution.

\section{Results}

Table 1 summarizes descriptive statistics for both the non-transformed and logarithm-transformed blood/ breath ratio data of Giguiere \& Simpson (7). Figure 1 depicts histograms with fitted normal curves for the actual (a) and ideal normal (b) distributions corresponding to the non-transformed data, with the latter figure reflecting the ideal normally distributed values from a distribution having the same mean and SD as the actual data. Figure 2 presents a similar depiction for the logarithm-transformed data. Both figures were generated by StatView 4.5 when it conducted the Kolmogorov-Smirnov normality test.

The non-transformed data are positively skewed (fig. 1a, $g_{1}=0.27$ ). A departure from normality due to skewness occurs barely at the $20 \%$ level of significance $(P \approx 0.20)$, given that the critical values of $g_{1}$ at the $2 \%$, $10 \%$, and $20 \%$ levels of significance are $\pm 0.49, \pm 0.34$, and \pm 0.26 , respectively. On the other hand, the kurtosis test shows this data to be more consistent with normality $\left(g_{2}=-0.42 ; g_{2}+3=2.58 ; P>0.20\right)$. The critical values of $\left(g_{2}+3\right)_{0.01,0.05,0.10}$ are, respectively, 2.26 , 2.42 , and 2.52 , and those of $\left(g_{2}+3\right)_{0.99,0.95,0.90}$ are, respectively, $4.19,3.68$, and 3.47 . The Geary kurtosis test, however, is apparently more sensitive in this case because the experimentally derived value of $a(0.8241)$ falls just outside the acceptance region at the $20 \%$ level of significance $(P \approx 0.20)$, indicating platykurtic behaviour. The critical values of $a_{0.01}, 0.05,0.10$ are, respectively, $0.7538,0.7678$, and 0.7751 , while the critical values of $a_{0.99,0.95,0.90}$ are, respectivively, 0.8410, 0.8299, and 0.8233 . 
The distribution of the logarithm-transformed data appears to be more symmetric on visual inspection, and more consistent with the Gaussian distribution based on the skewness test (fig. $2 \mathrm{a}, g_{1}=-0.14[P>0.20]$ ). The data do, however, deviate from normality in the direction of platykurtosis at the $20 \%$ level of significance $(P \approx 0.20)$, since the value of $g_{2}+3(2.48)$ barely falls outside the acceptance region at this level. Interestingly, the Geary kurtosis test statistic ( $a=0.8214)$ just makes it into the acceptance region $(P>0.20)$.

The arithmetic mean of the non-transformed data $(1868: 1)$ is not substantially different from the geometric mean $(1836: 1)$ stemming from the transformed data, with the two means related to each other via equation 4 $(1: 128)$. This equation yields an essentially unbiased estimate of $\overline{\mathrm{R}}$ - denoted by $E(\overline{\mathrm{R}})$ - from $\overline{\mathrm{R}}_{\mathrm{G}}$; the factor, 1.1513 , is equal to $(\ln 10) / 2$, and $[S D]^{2}$ is the sample variance in logarithmic units. The application of equation 4 to the relevant data in table 1 yields a value of $E(\overline{\mathrm{R}})$ equal to $1869: 1$, which is essentially identical to the value of $\bar{R}$ provided by StatView 4.5 .

$$
E(\overline{\mathrm{R}})=\operatorname{antilog}\left(\log \overline{\mathrm{R}}_{\mathrm{G}}+1.1513[\mathrm{SD}]^{2}\right)
$$

That the logarithmic transformation of the original data produces a distribution which more closely approximates the Gaussian distribution, based on visual inspection of figure $2 a$, can be gleaned from an examination of the individual CVs and medians. Normality is generally improved via logarithmic transformation when the CV exceeds $12 \%(1: 112)$, which is the case with the nontransformed data $(\mathrm{CV}=18.7 \%)$. Moreover, the greater symmetry of the distribution of the logarithm-transformed data can be ascertained by comparing its median (3.2653) to its mean (3.2639). The two are nearly identical, and the corresponding median $\mathrm{R}(1842: 1)$ and $\overline{\mathrm{R}}_{\mathrm{G}}$

Tab. 1 Descriptive statistics for $\mathrm{R}$ and $\log \mathrm{R}(n=137)$

\begin{tabular}{lll}
\hline Statistic & $\mathrm{R}$ & $\log \mathrm{R}$ \\
\hline Mean & $1868: 1$ & $3.2639^{\mathrm{a}}$ \\
$\mathrm{SD}$ & 349 & 0.0820 \\
$\mathrm{CV}$ & $18.7 \%$ & $2.5 \%$ \\
Minimum & $1190: 1$ & 3.0755 \\
Maximum & $2857: 1$ & 3.4559 \\
Range of Rs derived from mean \pm 2 SD & $1170: 1$ to $2566: 1$ & $1259: 1$ to $2679: 1$ \\
Relative error of mean \pm 2 SD compared to R $=2100: 1$ & $-44 \%$ to $+22 \%$ & $-40 \%$ to $+28 \%$ \\
$g_{1}$ (Skewness) & 0.27 & -0.14 \\
$g_{2}$; g +3 (Kurtosis) & $-0.42 ; 2.58$ & $-0.52 ; 2.48$ \\
(Geary kurtosis) & 0.8241 & 0.8214 \\
Kolmogorov-Sinirnov normality test: D and $P$ Values & $\mathrm{D}=0.051 ; P \approx 0.50$ & $\mathrm{D}=0.051 ; P \approx 0.50$ \\
Median & $1842: 1$ & 3.2653 \\
\hline
\end{tabular}

a Corresponds to $\log \overline{\mathrm{R}}_{\mathrm{G}}$, with $\overline{\mathrm{R}}_{\mathrm{G}}=1836: 1$.
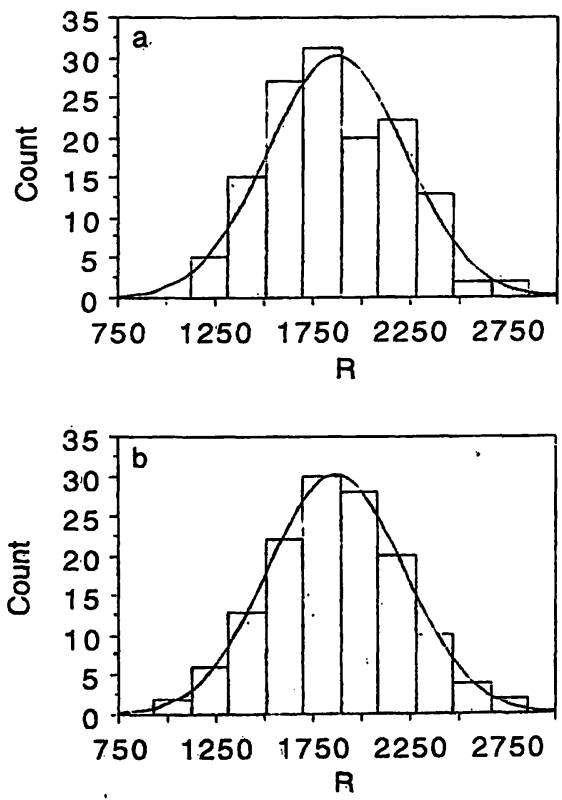

Fig. 1 Distribution histograms with fitted normal curves - generated via application of Kolmogorov-Smirnov normality test - for non-transformed blood/breath ratio data (a) of Giguiere \& Simpson (7) and for corresponding "ideal normal" data (b); blood/breath ratio denoted by $R$.
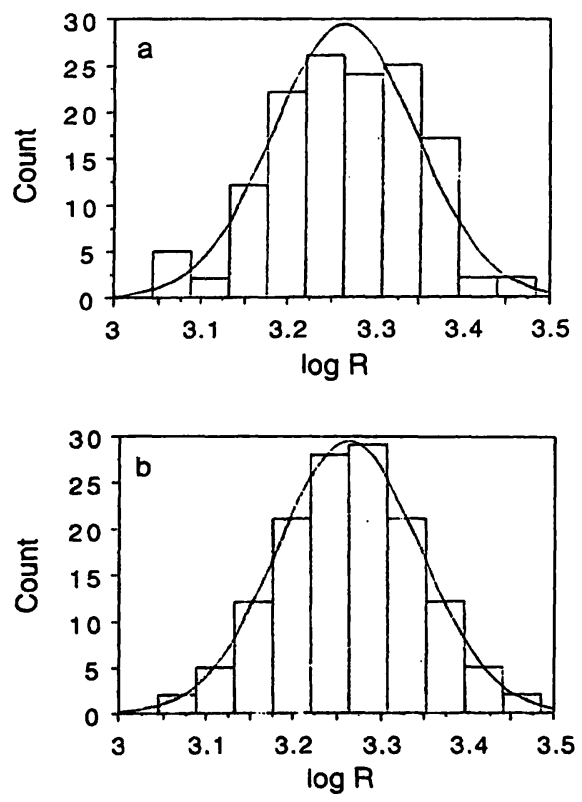

Fig. 2 Distribution histograms with fitted normal curves - generated via application of Kolmogorov-Smirnov normality test - for logarithm-transformed blood/breath ratio data (a) of Giguiere \& Simpson (7) and for corresponding "ideal normal" data (b); blood/ breath ratio denoted by $R$. 
(1836:1) differ by only $0.3 \%$. In this regard, 70 values of $\log R$ are above $\log \bar{R}_{G}$, and 67 values are below, compared to the first and last 68 values of $\log R$ that, respectively, lie above and below the value of $\log R$ (3.2653) corresponding to median $R$. In addition, while the minimum and maximum $R$ s are not uniformly distributed about $\bar{R}$ and deviate from $\bar{R}$ by -1.94 and $+2.83 \mathrm{SD}$, respectively, the minimum and maximum $\log$ Rs deviate from $\log \bar{R}_{\mathrm{G}}$ in considerably greater symmetrical fashion, given that the deviations are, respectively, -2.30 and $+2.34 \mathrm{SD}$. Figures $3 \mathrm{a}$ and $3 \mathrm{~b}$ illustrate the preceding points. These figures are univariate scattergrams of the values of $R$ and $\log R$, respectively, plotted along the horizontal axis in each case in the sequence they appear in their respective datasets. Display lines at the mean and the mean $\pm 2 \mathrm{SD}$ are also shown.

Despite the above differences regarding deviations from the mean for the non-transformed and logarithm-transformed data, the relative error ranges stemming from a comparison of the standard $2100: 1 \mathrm{blood} /$ breath ratio with $\bar{R} \pm 2 S D$ and with the Rs derived from $\log \bar{R}_{G}$ $\pm 2 \mathrm{SD}$, respectively, are not substantially different $(-44 \%$ to $+22 \%$ for the non-transformed data, and $-40 \%$ to $+28 \%$ for the logarithm-transformed data). This is not surprising when considered in the context of the results of the Kolmogorov-Smirnov normality test. For both sets of data, a value of $D$ of 0.051 was reported
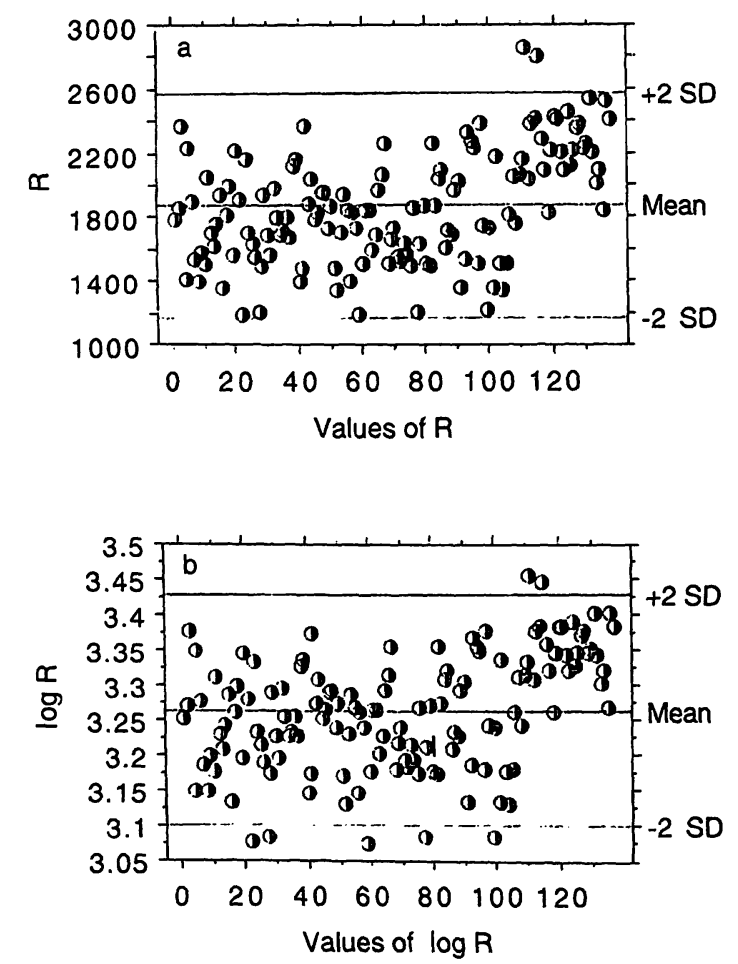

Fig. 3 Univariate scattergram of non-transformed blood/breath ratios (a) and corresponding logarithm-transformed ratios (b) reflecting data of Giguiere \& Simpson (7); blood/breath ratio denoted by $\mathrm{R}$; each value of $\mathrm{R}$ or $\log \mathrm{R}$ plotted along horizontal axis by StatView 4.5 in the sequence it appears in the dataset $(n=1$ to 137). by StatView 4.5 , indicating that neither dataset is rejected by the Kolmogorov-Smirnov test and that both are approximately normal $(P \approx 0.50)$. This $P$ value is consistent with critical values of $D$ associated with the application of the Kolmogorov-Smirnov test to samples from which the mean and SD must'be estimated, as was done for the datasets of this work $(17: 331,(19,20))$. Thus, logarithmic transformation did not result in a substantial improvement in the degree of normality of the non-transformed data.

\section{Discussion}

Given the apparent enhanced symmetry of the distribution of the logarithm-transformed data (fig. 2a) compared to the non-transformed data (fig. 1a), and the fact that the former generates statistical blood/breath ratio ranges that are more conservative than those generated by the latter, the following discussion concerning normal error analysis will focus primarily on the logarithmtransformed data. It must be emphasized, however, that normal error analysis can be applied to the non-transformed data as well, given the results of the Kolmogorov-Smirnov normality test cited previously. This, together with a consideration of Chebyshev's theorem $(17: 64,68,(21))$, which applies to any and all distributions of data values, permits a reasonably accurate parametric description of the logarithm-transformed data in terms of its $\mathrm{SD}$ and $\log \overline{\mathrm{R}}_{\mathrm{G}}$.

Chebyshev's theorem - after Russian mathematician $P$. L. Chebyshev (1821-1894) - can be stated as follows (21):

For any set of data (either population or sample) and for any constant $k$ greater than 1, the proportion of the data that must lie within $k$ standard deviations on either side of the mean is at least

$$
1-\frac{1}{k^{2}}
$$

Alternatively, the corresponding $P$ value for a particular sample, such as the data of this study, can be expressed in the form of Chebyshev's inequality $(17: 64)$ :

$$
P\left(\left|x_{i}-\bar{x}\right| \geq k \mathrm{SD}\right) \leq \frac{1}{k^{2}}
$$

Therefore, the minimal fraction of data falling within $2 \mathrm{SD}$ of the mean must be $75 \%(P \leq 0.25)$, and within $3 \mathrm{SD}, 88.9 \%(P \leq 0.11)$. Reference to figure $3 \mathrm{~b}$ indicates that, for the logarithm-transformed blood/breath ratio data addressed in this work, 130 of the 137 data values $(95 \%)$ lie within \pm 2 SD of $\log \bar{R}_{G}$ (corresponding Rs from table $1,1259: 1$ to $2679: 1$ ), which is obviously in accord with Chebyshev's.theorem and a distribution that is consistent with a Gaussian distribution. (For the less symmetrically distributed non-transformed 
data [fig. 3a], 135 of the 137 data values (98.5\%) lic within $\pm 1.94 \mathrm{SD}$ of $\overline{\mathrm{R}}$, with a corresponding range of $1190: 1$ to $2545: 1$ ). Furthermore, virtually all of the transformed data lie within $\pm 2.30 \mathrm{SD}$ of $\log \overline{\mathrm{R}}_{\mathrm{G}}$, as noted previously. Within the context of normality, this reflects essentially the central $98 \%$ of the population, with a corresponding range of Rs of $1190: 1$ to $2835: 1$, indicating a relative error range of $-43 \%$ to $+35 \%$, based on the standard $2100: 1$ ratio.

As emphasized by Rainey (22), $>95 \%$ certainty $(P<0.05)$ is the most common standard of proof used for assessing medical hypotheses, while in criminal proceedings, the well-accepted standard for scientific evidence, namely $>99 \%$ certainty $(P<0.01)$, would be more appropriate for meeting the "beyond-a-reasonabledoubt" requirement. This is essentially in agreement with the standard of $99.9 \%$ certainty $(P=0.001)$ reported by Jones (23) that is employed in Sweden for blood-alcohol concentration determinations based on direct blood analyses. The application of Rainey's argument, therefore, to the logarithm-transformed data of this work, and thus to the central $99 \%$ of those data (log $\bar{R}_{G} \pm 2.58 \mathrm{SD}$ ), would require minimal extrapolation of the range of Rs associated with the central $98 \%$ of the data. That extrapolation results in a range of $1128: 1$ to $2989: 1$, which is equivalent to an adjustment of the former range by about $\pm 5 \%$. The relative error range in this case, based on $2100: 1$, would be a nearly symmetrical $-46 \%$ to $+42 \%$.

While Heifer (10) determined blood/breath ratios for 133 subjects between 15 and $270 \mathrm{~min}$ after the end of drinking - using the evidential breath-alcohol analyzers, Alcotest 7010 (Draeger) and Alcomat (Siemens), an ethanol dose of $0.5-1.0 \mathrm{~g} / \mathrm{kg}$ bodyweight, and a consumption time of 10-90 min - he did not tabulate his data, as indicated previously. Nevertheless, estimates can be made from his graphed results which can be compared with the results obtained from the analysis of the non-transformed data of Giguiere \& Simpson (7). Heifer summarized his data in a plot of $\mathrm{BrAC} / \mathrm{BAC} v$ time after the end of drinking (fig. 1 of his article), so that, based on the standard $2100: 1$ ratio, a value of $R$ can be estimated from this plot by calculating the product of 2100 and the reciprocal of a relevant $\mathrm{BrAC} / \mathrm{BAC}$ value. $\mathrm{He}$ included values of $\bar{R}$ reflecting a total of 1150 paired blood and breath specimens - which represent the greatest number of blood/breath pairs ever reported in a laboratory study - at 15 (119 pairs), 30 (116 pairs), 60 (109 pairs), 90 (103 pairs), 120 (102 pairs), 150 (133 pairs), 180 (132 pairs), 210 (113 pairs), 240 (108 pairs), and $270 \mathrm{~min}$ (115 pairs) after the end of drinking. Also included at each of these times were the data points corresponding to $\bar{R} \pm 2 \mathrm{SD}$. Since 136 of the 137 measurements analyzed in our work were obtained between 15 and $124 \mathrm{~min}$ after the end of drinking, and since omis- sion of the 137 th measurement $(2415: 1)$ taken at 179 min would have had virtually no effect on the results generated from statistical analysis of the overall data, estimates made from Heifer's work for comparison with our results were restricted to his $15-$ to 120 -min time frame. Under these conditions, the following estimates can be made from figure 1 of Heifer's article (It should be noted that Heifer confirmed - via written communication to Simpson in May, 1993 - our interpretation of and the estimates made from figure 1, and that his blood/ breath ratio data conform closely to a Gaussian distribution.): $\overline{\mathrm{R}} \approx 1780: 1 ; \mathrm{SD} \approx 350 ; \mathrm{CV} \approx 20 \% ; \overline{\mathrm{R}} \pm 2 \mathrm{SD}$ $\approx 1080: 1$ to $2480: 1$ (relative error range, based on $2100: 1:-49 \%$ to $+18 \%)$.

A comparison of these results with those derived from the non-transformed data of Giguiere \& Simpson (7) indicates that the latter are more conservative, but not substantially so, given that the corresponding relative error range for the Giguiere/Simpson data is $-44 \%$ to $+22 \%$. Moreover, since the $\mathrm{CV}$ associated with Heifer's data exceeds $12 \%$, logarithmic transformation would be expected to improve the normality of the distribution of that data $(1: 112)$. Nevertheless, the message stemming from the above analysis of Heifer's data is consistent with the message of this work, which is summarized in the following conclusion.

\section{Conclusion}

As has been reported earlier $(8-11)$, the magnitude of the blood/breath ratio is dependent on the time elapsed after the end of drinking. Nearly all of the data analyzed in this work ( $99.3 \%$, or 136 of 137 measurements) were obtained between 0.25 and $2.07 \mathrm{~h}$ after the end of drinking. This period has been characterized as the absorptive/ plateau phases of alcohol metabolism, or the time required to reach peak blood-alcohol concentration (11). In this regard, Baselt \& Danhof (24) reported that, for fasting subjects, 0.5 to $2.1 \mathrm{~h}$ after the end of drinking must elapse before peak blood-alcohol concentration is reached. Certainly the factor of food consumption, including the type and quantity of food eaten, would contribute to an extension of both the lower and upper bounds of these ranges, as confirmed by Baselt \& Danhof (24). For non-fasting subjects, they specified a timeto-peak blood-alcohol concentration range of 1.0 to $6.0 \mathrm{~h}$. In addition, Dubowski (11) summarized data from his experiments conducted on both female and/or male subjects that were consistent with the conclusions of $\mathrm{Ba}$ selt \& Danhof. Dubowski also emphasized that, in addition to the factor of food consumption, the rate of alcohol absorption is dependent on other factors, including the type and concentration of alcoholic beverage ingested, and a "multitude of other physical, biological, psychological and time factors ... [and] the individual's 
sex, body weight and body water, and related habitus characteristics as well as offsetting metabolic disposition."

Under field conditions, the driving-while-intoxicated arrestee's absorption status at the time of the breath test is generally unknown. Clearly, the time frame $0.25-2 \mathrm{~h}$ after the end of drinking is important, although it is often difficult to identify the point in this time frame characterizing a particular arrestee when his/her breath test is administered. Therefore, as emphasized below, this overall general time frame is best used to characterize the absorption status of a driving-while-intoxicated arrestee when that status is unknown. Nevertheless, given the dependence of the blood/breath ratio on the time elapsed after the end of drinking, figure 1 of Heifer's article (10), which reflects non-transformed data that is clearly more extensive than the corresponding data of this work, can be used to assess the variability of the blood/breath ratio according to the five specific times comprising Heifer's $0.25-2 \mathrm{~h}$ time frame. Thus, his estimated values of $\bar{R}$ and associated parenthetical values of SD at 15,30,60,90, and $120 \mathrm{~min}$ after the end of drinking are, respectively, $1365: 1$ (320); 1680:1 (365); $1910: 1$ (365); $1945: 1$ (355); $2020: 1$ (330).

Whenever it can be established by objective means that a driving-while-intoxicated arrestee is "fully postabsorptive," then the blood/breath ratio data summarized by Dubowski (11), which reflect the results of a study he conducted with O'Neill (25) on healthy, "fully postabsorptive" males, may be applicable. (In this regard, $D u$ bowski [11] did not thoroughly define the label, "fully postabsorptive"; that is, he did not specify how much time after peak blood-alcohol concentration was required before subjects were deemed fully postabsorptive, nor did he specify the blood-alcohol or breath-alcohol concentrations involved.) That study produced an $\bar{R}$ of $2280: 1$ ( $\mathrm{SD}=241.5, \mathrm{CV}=10.6 \%$ ) derived from analysis of 393 paired blood and breath specimens. Dubowski stated explicitly that the data "have a Gaussian distribution" and reported a range of $1555: 1$ to $3005: 1$ ( $\pm 3 \mathrm{SD}$ ) for $99.7 \%$ of the population (relative error range, based on $2100: 1:-26 \%$ to $+43 \%$ ). For $99 \%$ of the population ( $\pm 2.58 \mathrm{SD}$ ), as per Rainey's stipulation
(22), the range would be narrowed slightly to $1657: 1$ to $2903: 1$ (relative error range: $-21 \%$ to $+38 \%$ ). Logarithm-transformation of this data would not be expected to result in a significant improvement in normality because, given a $\mathrm{CV}$ of $10.6 \%$, such improvement, as noted previously, generally occurs when the $\mathrm{CV}$ exceeds $12 \%((1)$, p. 112$)$.

If, on the other hand, a driving-while-intoxicated arrestee's absorption status is unknown, and he/she is to be given the benefit of the doubt, then the results of the present work are applicable, as indicated previously. At the very minimum, the relative error range listed in table 1 for the range of Rs derived from $\log \bar{R}_{G} \pm 2 \mathrm{SD}$ ( $-40 \%$ to $+28 \%$, based on $1259: 1$ to $2679: 1$ ) should apply, and for $99 \%$ of the population, reflecting $\log \bar{R}_{G}$ $\pm 2.58 \mathrm{SD}$, the applicable relative error range would be $-46 \%$ to $+42 \%$, based on $1128: 1$ to $2989: 1$.

In the final analysis, the recommendations offered here are consistent with Dubowski's assessment of the generally accepted mean postabsorptive blood/breath ratio of approximately $2300: 1$ (11): "significant variations from this population mean exist during active alcohol absorption and in some individuals even in the postabsorptive phase." This article quantifies the errors produced by such variations when estimates of blood-alcohol concentration are made by means of breath-alcohol analysis. Moreover, the statistical analysis presented here indicates there is little merit to the claim that, because of skewing, it is inappropriate to apply normal error analysis to blood/breath ratios to estimate error limits at the 95 and $99 \%$ confidence levels. In this regard, while use of a logarithmic transformation of blood/ breath ratio data is the basis for the principal conclusions of this article, the results of this work indicate that the non-transformed data can also provide useful estimates of the amount of error expected in breath test results.

\section{Acknowledgements}

The authors thank Professor Terence P. Speed of the Department of Statistics, University of California (Berkeley), for reviewing the manuscript and providing us with his expert assistance concerning the application of the Kolmogorov-Smirnov normality test and other statistical issues addressed in this article.

\section{References}

1. Bliss CI. Statistics in biology. Vol. 1. New York: McGrawHill, 1967.

2. Gaddum JH. Lognormal distributions. Nature 1945; $156: 463-6$.

3. Jones AW. Dilemma of a constant blood/breath ratio of ethanol in chemical test evidence of intoxication. In: Alcohol, drugs \& traffic safety. Proceedings of the T89 11th International Conference on Alcohol, Drugs and Traffic Safety; 1989 Oct 24-27; Chicago. Chicago (IL): National Safety Council, 1990:237-42.

4. Galton F. The geometric mean, in vital and social statistics. Proc Roy Soc 1879; 29:365-7.

5. Aitchison J, Brown JAC. The lognormal distribution. Cambridge: Cambridge University Press, 1957.

6. Finney DJ. Statistical method in biological assay. 2nd ed. 'Charles Griffin \& Co.: London, 1964:38-9.

7. Giguiere W, Simpson G. Medicolegal alcohol determination: in vivo blood/breath ratios as a function of time, I. In: McLinden VJ, Haney DJ, editors. Proceedings of the 27th Meeting of the International Association of Forensic Toxicologists 1990 
Oct 19-23; Perth, Australia. Perth, Australia: International Association of Forensic Toxicologists, 1992:494-506.

8. Alobaidi TAA, Hill DW, Payne JP. Significance of variations in blood : breath partition coefficient of alcohol. Brit Med J 1976; 2:1476-81.

9. Jones AW. Variability of the blood : breath alcohol ratio in vivo. J Stud Alc 1978; 39:1931-9.

10. Heifer U. Atemalkoholkonzentration/Blutalkoholkonzentration: Utopie eines forensisch brauchbaren Beweismittels. Blutalkohol 1986; 23:229-38.

11. Dubowski KM. Absorption, distribution, and elimination of alcohol: highway safety aspects. J Stud Alc 1985; Suppl 10:98-108.

12. Dubowski KM. Recent developments in alcohol analysis. Alc Drugs Driving 1986; 2:13-46.

13. Geary RC. Moments of the ratio of the mean deviation to the standard deviation for normal samples. Biometrika 1936; 28:295-307.

14. Remington RD, Schork MA. Statistics with applications to the biological and health sciences. Englewood Cliffs, NJ: PrenticeHall, 1970:223.

15. Pearson K, Hartley HO, editors. Biometrika tables for statisticians. 3rd ed., Vol. 1. London: Bentley House, 1966:207, Table $34 \mathrm{~A}$.

16. Snedecor GW, Cochran WG. Statistical methods. 6th ed. Ames, IA: Iowa State University Press, 1967.

17. Sachs L. Applied statistics: a handbook of techniques. New York: Springer-Verlag, 1982.
18. Baron C, Haycock KA, Schorer P. QC tools for Stat View: statistical process control tools for Statview. Berkeley (CA): Abacus Concepts, 1993:83-5.

19. Massey FJ Jr. The Kolmogorov-Smirnov test for goodness of fit. J Amer Stat Assoc 1951; 46:68-78.

20. Lilliefors HW. On the Kolmogorov-Smirnov test for normality with mean and variance unknown. J Amer Stat Assoc 1967; 62:399-402.

21. Brase $\mathrm{CH}$, Brase CP. Understandable statistics: concepts and methods. 4th ed. Lexington, MA: DC Heath, 1991:85.

22. Rainey PM. Relation between serum and whole-blood ethanol concentrations. Clin Chem 1993; 39:2288-92.

23. Jones AW. Differences between capillary and venous bloodalcohol concentrations as a function of time after drinking, with emphasis on sampling variations in left vs right arm. Clin Chem 1989; 35:400-4.

24. Baselt RC, Danhof IE. Disposition of alcohol in man. In: Garriott JC, editor. Medicolegal aspects of alcohol determination in biological specimens. Littleton, MA: PSG Publishing, 1988:55-73.

25. Dubowski KM, O'Neill B. The blood/breath ratio of ethanol. Clin Chem 1979; 25:1144.

Received May 20/October 31, 1995

Corresponding authors: Professor Dominick A. Labianca, Department of Chemistry, Brooklyn College of The City University of New York, Brooklyn, New York 11210, USA, Dr. G. Simpson, P.O.Box 1551, Thousand Oaks, CA 91358, USA 
